\section{(2) OPEN ACCESS}

\title{
Synovial tissue signatures enhance clinical classification and prognostic/treatment response algorithms in early inflammatory arthritis and predict requirement for subsequent biological therapy: results from the pathobiology of early arthritis cohort (PEAC)
}

\author{
Gloria Lliso-Ribera, ${ }^{1}$ Frances Humby $\odot,{ }^{2}$ Myles Lewis $\odot,{ }^{3}$ Alessandra Nerviani $\odot,{ }^{1}$ \\ Daniele Mauro, ${ }^{1}$ Felice Rivellese $\odot{ }^{1}$, Stephen Kelly, ${ }_{1}$ Rebecca Hands, ${ }^{5}$ Fabiola Bene, ${ }^{3}$ \\ Nandhini Ramamoorthi, ${ }^{6}$ Jason A Hackney, ${ }^{7}$ Alberto Cauli, ${ }^{8}$ Ernest H Choy, ${ }^{9}$ \\ Andrew Filer, ${ }^{10}$ Peter C Taylor $\odot,{ }^{11}$ lain Mclnnes, ${ }^{12}$ Michael J Townsend, ${ }^{7}$ \\ Costantino Pitzalis (1) $^{13}$
}

\section{Handling editor Josef S \\ Smolen}

For numbered affiliations see end of article.

\section{Correspondence to}

Professor Costantino Pitzalis, Experimental Medicine and Rheumatology, William Harvey Research Institute, London EC1M 6BQ, UK c.pitzalis@qmul.ac.uk

GL-R and FH contributed equally.

Received 20 May 2019 Revised 26 August 2019 Accepted 27 August 2019 Published Online First 2 October 2019
Check for updates

(c) Author(s) (or their employer(s)) 2019. Re-use permitted under CC BY. Published by BMJ.

To cite: Lliso-Ribera $\mathrm{G}$,

Humby F, Lewis M,

et al. Ann Rheum Dis

2019:78:1642-1652.

\section{ABSTRACT}

Objective To establish whether synovial pathobiology improves current clinical classification and prognostic algorithms in early inflammatory arthritis and identify predictors of subsequent biological therapy requirement. Methods 200 treatment-naïve patients with early arthritis were classified as fulfilling RA1987 American College of Rheumatology (ACR) criteria (RA1987) or as undifferentiated arthritis (UA) and patients with UA further classified into those fulfilling RA2010 ACR/European League Against Rheumatism (EULAR) criteria. Treatment requirements at 12 months (Conventional Synthetic Disease Modifying Antirheumatic Drugs (csDMARDs) vs biologics vs no-csDMARDs treatment) were determined. Synovial tissue was retrieved by minimally invasive, ultrasound-guided biopsy and underwent processing for immunohistochemical (IHC) and molecular characterisation. Samples were analysed for macrophage, plasma-cell and B-cells and T-cells markers, pathotype classification (lympho-myeloid, diffuse-myeloid or pauci-immune) by IHC and gene expression profiling by Nanostring.

Results 128/200 patients were classified as RA1987, 25 as RA2010 and 47 as UA. Patients classified as RA1987 criteria had significantly higher levels of disease activity, histological synovitis, degree of immune cell infiltration and differential upregulation of genes involved in B and T cell activation/function compared with RA2010 or UA, which shared similar clinical and pathobiological features. At 12-month follow-up, a significantly higher proportion of patients classified as lympho-myeloid pathotype required biological therapy. Performance of a clinical prediction model for biological therapy requirement was improved by the integration of synovial pathobiological markers from $78.8 \%$ to $89 \%-90 \%$.

Conclusion The capacity to refine early clinical classification criteria through synovial pathobiological markers offers the potential to predict disease outcome and stratify therapeutic intervention to patients most in need.

\section{Key messages}

What is already known about this subject?

- The introduction of ACR/EULAR rheumatoid arthritis (RA) classification criteria has impacted positively on early diagnosis and treatment of RA leading to better outcomes. By the same token, broader criteria have led to the inclusion of patients with milder and more heterogenous disease. This, together with the inability to precisely predict disease prognosis and treatment response at the individual patient levels, emphasise the need to identify patients at risk of accelerated structural damage progression and fast-track aggressive/biological therapies to patients with poor prognosis.

\section{INTRODUCTION}

The introduction of new classification criteria for rheumatoid arthritis (RA) in $2010^{1}$ has been demonstrated to be clinically useful with enhanced diagnostic sensitivity in early disease compared with 1987 criteria $^{2}$; however, this is balanced by a lower specificity. ${ }^{34}$ This is of particular importance, as data suggest that approximately $40 \%$ of patients with early inflammatory arthritis, not fulfilling 1987 criteria, may spontaneously remit while approximately $30 \%$ will progress to RA. ${ }^{5}$ Critically, the mechanisms underlying the transition from undifferentiated arthritis (UA) to RA remain unknown though it has been suggested that qualitative or quantitative difference within synovial tissue may contribute to diverse disease evolution and/or treatment response. ${ }^{6} 7$ Thus, pretreatment stratification of early inflammatory arthritis is important in order to target therapy to poor prognosis patients. Previous data suggest that stratifying early arthritis according to RA2010 versus RA1987 classification criteria reveals significant clinical heterogeneity in diagnosis at 2-year 
Key messages

\section{What does this study add?}

- This study analyses the largest biopsy-driven early inflammatory arthritis cohort to date (200 patients) and, through a detailed synovial cellular and molecular characterisation refines ACR/EULAR disease classification. In addition, the study identifies synovial pathobiological markers associated with the lympho-myeloid pathotype and the requirement of biological therapy at 12 months, reinforcing recently published data that indicate that these patients are affected by highly aggressive disease and worse radiographic outcome. Notably, these findings are independent from the time of diagnosis within the first 12 months of symptoms initiation, suggesting that the socalled 'window of opportunity' is wider than 6 months and early stratification of biological therapies according to poor prognostic synovial pathobiological subtypes at disease onset may improve the outcome of these patients. The integration of synovial pathobiological markers into a logistic regression model improves the prediction accuracy from $78.8 \%$ (clinical) to $89 \%-90 \%$ (clinical +molecular) and enables the identification at disease onset of patients who subsequently require biological therapy. Thus, this study provides support to the notion that biological therapies should be started early in patients with poor prognosis.

\section{How might this impact on clinical practice or future developments?}

- The identification at disease onset of patients who are unlikely to respond to csDMARDs remains a major unmet need. The capacity to refine early clinical classification criteria through the application of synovial pathobiological markers and the ability to identify patients who subsequently require biological therapy at disease onset offer the opportunity to stratify therapeutic intervention to the patients most in need. This present study adds weight to the need to change current therapeutic algorithms and start biological therapies at disease onset in patients with poor prognosis. This is likely to have a major impact on disease control/remission and longterm disability, as notionally supported by numerous early intervention studies using biological therapies.

follow-up ${ }^{8}$ although subsequent analysis of synovial tissue did not suggest that such clinical heterogeneity translated to significant differences in synovial pathobiology. ${ }^{9}$ However, recently published data from a cohort of 144 patients with early RA have demonstrated that synovial cellular and molecular signatures define prognostic and treatment response phenotypes. ${ }^{10}$ Importantly, whether clinical heterogeneity associated with the introduction of the 2010 ACR/EULAR criteria can be explained by synovial pathobiological signatures and whether they associate with subsequent disease outcome, up to now, remain unknown.

Therefore, the aim of this study was to investigate whether in patients with early inflammatory arthritis synovial cellular and molecular signatures: (1) segregate according to clinical classification (RA1987 vs RA2010 vs UA), (2) change depending on symptom duration and (3) determine prognosis including subsequent requirement for biological therapy.

\section{PATIENTS AND METHODS}

\section{Patients}

Two hundred consecutive patients with inflammatory arthritis recruited at Barts Health NHS Trust as part of the multicentre pathobiology of early arthritis cohort (http://www.peac-mrc. mds.qmul.ac.uk) were included within the study. Patients were treatment naïve (csDMARD and steroid) and had <1year symptoms.

At baseline, patients underwent collection of routine demographic data and were categorised according to the following criteria: (1) RA1987 ${ }^{2}$ or (2) UA. 2010 ACR/EULAR criteria for $\mathrm{RA}^{1}$ were then applied to further classify patients with UA, resulting in three groups: (1) RA1987 (RA1987+/RA2010+), (2) RA2010 (RA1987-/RA2010+) and (3) UA (RA1987-/ RA2010-). An ultrasound-guided synovial biopsy of a clinically active joint was performed. ${ }^{11}$ Patients were then given standard conventional synthetic csDMARD therapy with a treat-to-target approach to treatment escalation (Disease Activity Score 28 joints (DAS28) <3.2). Patients failing csDMARD therapy were given biological therapy (anti-tumour necrosis factor (TNF), tocilizumab or rituximab) according to the prevailing UK National Institute for Clinical Excellence (NICE) prescribing algorithm if they continued to have a DAS28 $>5.1$ following 6 months of therapy. ${ }^{12}$ At 12-month, follow-up patients were categorised as follows: (1) self-limiting (SL) disease (DAS28<3.2 and off csDMARD/steroid therapy) versus persistent disease (PD) (DAS28 > 3.2 and/or csDMARD) and (2) symptomatic treatment (non-steroidal anti-inflammatories) versus csDMARD therapy versus biological \pm csDMARD therapy.

\section{Synovial biopsy collection and processing}

A minimum of six biopsies per patient were collected for paraffin embedding and if intact lining layer identified underwent histopathological assessment. Synovitis score was determined using a previously validated scoring system. ${ }^{13}$ Following immunohistochemical staining of sequentially cut slides using previously reported protocols for B cells (CD20), T cells (CD3), macrophages (CD68) and plasma cells (CD138), the degree of immune cell infiltration was assessed semiquantitatively (0-4). ${ }^{14}$ Biopsies were stratified into one of three synovial pathotypes according to the following criteria: (1) lympho-myeloid presence of grade 2-3 CD20 +aggregates, (CD20 $\geq 2)$ and/or CD138 $>2$, (2) diffuse-myeloid CD68 SL $\geq 2$, CD20 $\leq 1$ and/or CD3 $\geq 1$, CD138 $\leq 2$ and (3) pauci-immune CD68 SL $<2$ and CD3, CD20, CD138 <1.

\section{Nanostring analysis}

A minimum of six synovial samples per patient were immediately immersed in RNA-Later and RNA extraction performed as previously described. ${ }^{10}$ RNA samples then underwent profiling for the expression of 238 genes preselected based on previous microarray analyses of synovial tissue from patients with established $\mathrm{RA}^{15}$ and/or relevance to RA pathogenesis. Raw NanoString counts were processed using the NanoStringQCPro package in R V.3.2.0. Counts were normalised for RNA content by global gene count normalisation and then $\log$ transformed (base 2). The validity of normalisation was then checked via box plot and scatter plot of normalised counts. Benjamini-Hochberg method was used to adjust for multiple testing, and genes were considered to be differentially expressed if they demonstrated an false discovery rate (FDR)adjusted $\mathrm{p}<0.01$. 


\section{Statistical analysis}

Statistical analyses were run using R V.3.0.2. For three-way comparisons, the Kruskal-Wallis test was used for continuous and $\chi^{2}$ or Fisher's exact test used for categorical variables as appropriate. A p $<0.05$ was considered statistically significant. Post hoc comparison tests were performed using the Dunn test or Bonferroni correction as appropriate.

\section{Linear regression models}

Logistic regression using forward, backward and bidirectional stepwise selection was employed using the glm function in R.

Gene expression predictors were selected by L1 (LASSO) sparse logistic regression using $\mathrm{R}$ package glmnet. The penalty parameter $\lambda$ was optimised using 10 -fold cross validation. $\lambda$ corresponding to the minimum mean cross-validated error was retained as final penalty parameter in the model.

\section{Predictive performance evaluation}

Predictive performance of the final prediction model was assessed by computing the area under the receiver operating characteristic curve (AUC), using both apparent and internal validation with 95\% CI. Internal validation using a bootstrap method $^{1617}$ (performed with $\mathrm{R}$ package boot version 1.3-18) was employed to correct for overfitting, to generate unbiased optimism-adjusted estimates of the C statistic (AUC) with low absolute error. Bootstrap estimate of the AUC statistic was computed by random sampling with replacement 500 times to enable estimation of the optimism corrected AUC.

\section{RESULTS}

\section{Patient demographics and clinical correlations}

Two hundered pathobiology of early arthritis cohort patients were included: 128/200 (64\%) patients were classified as RA1987 (RA1987+/RA2010+) and 72/200 (36\%) as UA. Of the patients with UA, 25 were further classified as RA2010 (RA1987-/ RA2010+) (25/200, 12.5\%) and 47 remained as UA (RA1987-/ RA2010-) (47/200, 23.5\%) (figure 1A). No significant difference in mean age, disease duration or erythrocyte sedimentation rate (ESR) between groups was demonstrated. However, the RA1987 group had significantly higher levels of $\mathrm{C}$ reactive protein (CRP), tender joint count (TJC), swollen joint count (SJC), DAS28, rheumatoid factor (RF), anticitrullinated protein antibody (ACPA) and Visual Analogue Score (VAS) and significantly higher numbers of patients seropositive for RF and ACPA compared with either the RA2010 or UA groups (figure 1B). SJC and ACPA titres were the only clinical parameters with significant differences between the RA2010 and UA groups, indicating that in terms of clinical measures of disease activity these two groups are relatively homogenous.

\section{Synovial pathotypes distinguish clinical phenotypes regardless of disease duration}

Synovial biopsies were obtained predominantly from small joints $(81.5 \%)$ (figure 2A). Patients with synovial tissue suitable for histological analysis (166/200) were segregated according to baseline synovial pathotype (figure $2 \mathrm{~B}$ ) and differences in clinical parameters evaluated. We demonstrated significantly higher mean DAS28 within the lympho-myeloid compared with either the diffuse-myeloid or pauci-immune group (5.82 vs 4.93 vs 4.86 , $\mathrm{p}<0.001)$. Mean CRP was significantly higher in the lymphomyeloid and diffuse-myeloid versus pauci-immune groups (16.86 vs 15.52 vs $9.55, \mathrm{p}<0.001)$ and a significantly higher number of patients were seropositive for either RF $(p=0.012)$ or ACPA ( $p=0.011$ ) within the lympho-myeloid group (figure $2 \mathrm{C}$ ). To evaluate whether disease duration influenced prevalence of synovial pathotype, patients were stratified into four groups according to disease duration at baseline $(1-3 \mathrm{~m}, 4-6 \mathrm{~m}, 7-9 \mathrm{~m}$ and $10-12 \mathrm{~m}$ ) and frequency of synovial pathotype determined. No significant differences in synovial pathotype frequency at each timepoint were demonstrated $(p=0.65)$ (figure 2D).

RA1987 patients display significantly higher levels of synovial immune cell infiltration compared to RA2010 and UA patients Patients were segregated according to pathotype and further into RA1987, RA2010 and UA categories. A higher proportion of patients within the RA1987 group were categorised as lymphomyeloid (vs diffuse myeloid or pauci-immune) (43.5\% vs 33\% vs 23.5\%) (figure 3A). We also demonstrated a significantly higher mean synovitis, CD3 + T cell, CD20 + B cell, CD138 + plasmacell and CD68 +SL/L macrophage score between the RA1987 group and both the RA2010 and UA groups $(\mathrm{p}<0.001)$ (figure 3B). We saw no significant differences in synovitis score, mean CD3 $+\mathrm{T}$, CD20 + B, CD68 + Lor SL macrophage or CD138 + plasmacell number between the RA2010 and UA group (figure 3B), indicating that these two groups are relatively homogenous in terms of tissue pathology.

\section{Synovial genes regulating B cell activation and function are significantly upregulated in RA1987 patients compared to the RA2010/UA groups}

Of 200 patients, 145 had RNA available for Nanostring analysis (95/128 RA1987 patients, 12/25 RA2010 patients and $38 / 47$ patients with UA) and were analysed for differential gene expression (238 genes) between groups.

Comparing RA1987 versus RA2010 groups, we demonstrated a significant differential expression of 53 genes (figure 3C). In line with the histological analysis, a number of differentially upregulated genes within the RA1987 cohort were involved in mediating B cell activation/function (eg, CD79A, CD38, IGJ, CXCL13, IRF4, CCL19, CD38, TNFA and IL6). When evaluating gene expression between RA1987 and UA groups, we found a similar trend with differential upregulation of a number of genes within the RA1987 cohort mediating B cell activation/function although only CXCL13 remained significant following correction for multiple comparisons (figure 3D). Conversely, when evaluating gene expression between the RA2010 and UA cohorts, only seven genes appeared as significant with a preponderance of differentially upregulated genes within the RA2010 cohort mediating cartilage biology (COMP, $D K K 3, I N H B A)$ and none remaining significant after correction for multiple comparisons (figure $3 \mathrm{E}$ ).

\section{Classification as RA1987 criteria at disease onset predicts PD at 12 months}

Of 200 patients, 190 had 12-month follow-up data available; we examined whether baseline synovial pathotype was associated with disease evolution. 119/121 (99\%) RA1987 patients and 19/22 (90\%) RA2010 had PD (figure 4A). Within the UA cohort, 11/47 (23\%) had other diagnoses. Of the remaining 36 patients, 26/36 (72.2\%) had PD, and 10/36 (27.8\%) SL. Of the patients with UA with PD, 4/26 (15.3\%) progressed to fulfil 2010 ACR/EULAR criteria RA at 12 months. Results demonstrated a significantly higher proportion of patients with SL disease in the UA group compared with the RA2010 or RA1987 groups and a significantly higher number of patients within the RA1987 group with PD (figure 4B). When evaluating the effect of baseline pathotype, we demonstrated a higher proportion of patients with a lympho-myeloid versus diffuse-myeloid or pauci-imune pathotype (39\% vs $32 \%$ vs $13 \%$ ) with $\mathrm{PD}$ and a higher number of patients with a diffuse-myeloid versus 
A

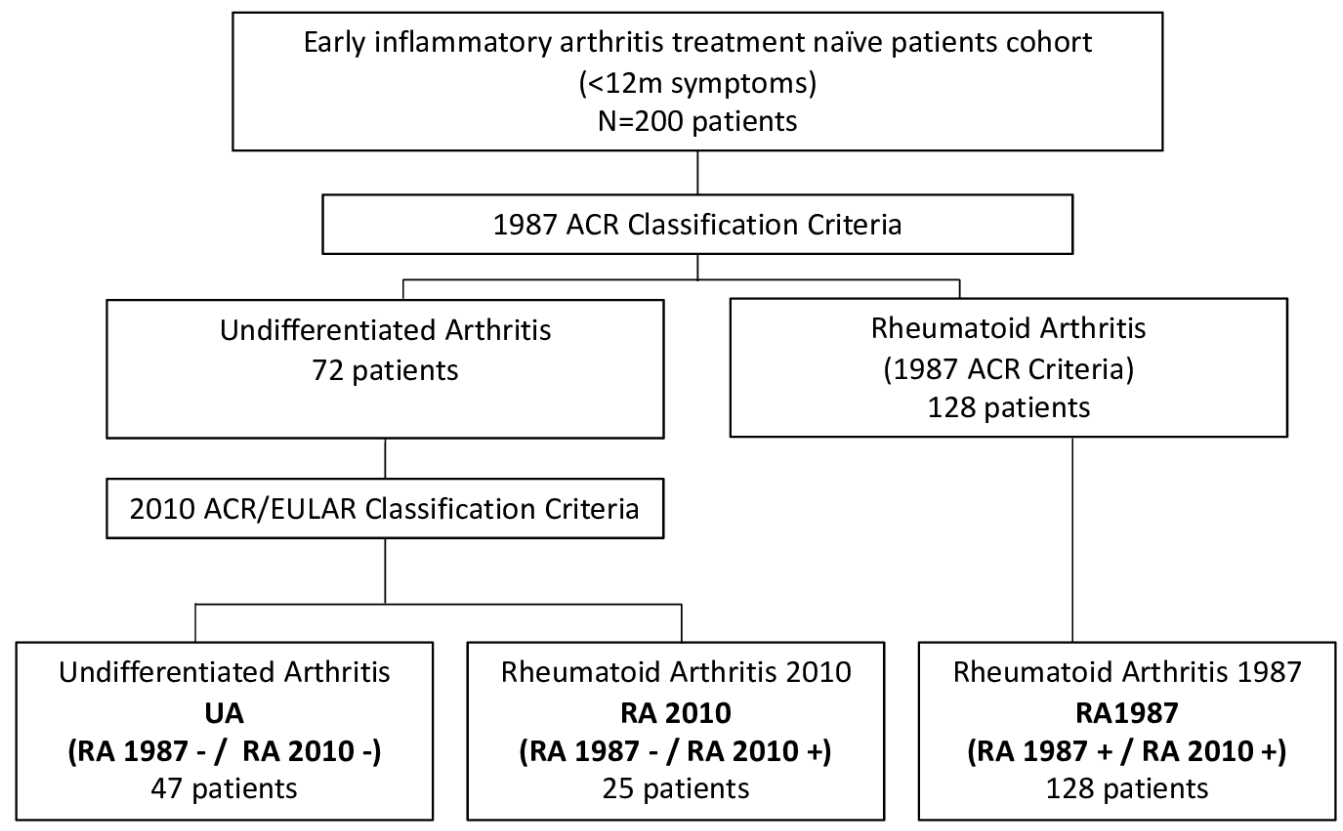

B

\begin{tabular}{|c|c|c|c|c|c|c|c|}
\hline N 200 & $\begin{array}{c}\text { RA } 1987 \\
\text { (RA } 1987+\text { ) } \\
\text { RA 2010 +) } \\
\text { N } 128\end{array}$ & $\begin{array}{c}\text { RA } 2010 \\
\text { (RA } 1987 \text { - / } \\
\text { RA 2010+) } \\
\text { N } 25\end{array}$ & $\begin{array}{c}\text { UA } \\
\text { (RA1987 - / } \\
\text { RA2010-) } \\
\text { N } 47\end{array}$ & p-value & $\begin{array}{l}\text { p-value } \\
\text { (post-hoc) } \\
\text { RA 1987- } \\
\text { UA }\end{array}$ & $\begin{array}{c}\text { p-value } \\
\text { (post-hoc) } \\
\text { RA1987- } \\
\text { RA2010 }\end{array}$ & $\begin{array}{l}\text { p-value } \\
\text { (post-hoc) } \\
\text { RA2010- } \\
\text { UA }\end{array}$ \\
\hline Age (years). Mean (SD) & $52.64(16.02)$ & $52.25(12.54)$ & $52.76(15.33)$ & 0.98 & & & \\
\hline Disease duration (months). Mean (SD) & $5.64(4.48)$ & $10.47(25.28)$ & $6.11(3.51)$ & 0.91 & & & \\
\hline ESR. Mean (SD) & $39.05(19.69)$ & $30.64(30.06)$ & $10.63(21.51)$ & 0.56 & & & \\
\hline CRP. Mean (SD) & $17.82(13.89)$ & $14.6(20.36)$ & $7.21(12.35)$ & $0.03 *$ & $<0.001 *$ & 0.12 & 0.071 \\
\hline 28 TJC. Mean (SD) & $11.98(7.29)$ & $6.88(5.72)$ & $6.80(6.79)$ & $<0.001^{*}$ & $<0.001^{*}$ & $0.0012^{*}$ & 0.74 \\
\hline 28 SJC. Mean (SD) & $7.68(5.62)$ & $5.68(4.91)$ & $3.10(2.82)$ & $<0.001 *$ & $<0.001 *$ & $0.042 *$ & $0.031 *$ \\
\hline Das 28. Mean (SD) & $5.76(1.35)$ & $4.73(1.56)$ & $4.001(1.51)$ & $<0.001 *$ & $<0.001 *$ & $0.002 *$ & 0.13 \\
\hline Vas global disease activity. Mean (SD) & $64.82(24.80)$ & $45.36(28.78)$ & $34.55(29.27)$ & $<0.001^{*}$ & $<0.001 *$ & $0.0043 *$ & 0.17 \\
\hline RF titre. Mean (SD) & $25.53(22.49)$ & $2.68(2.95)$ & $1.27(1.42)$ & $<0.001 *$ & $<0.001 *$ & $<0.001^{*}$ & 0.21 \\
\hline ACPA titre. Mean (SD) & $26.16(18.42)$ & $75.24(175.40)$ & $1.68(10.56)$ & $<0.001 *$ & $<0.001 *$ & $<0.001 *$ & $0.01 *$ \\
\hline RF +ve. N (\%) & $84(65 \%)$ & $7(28 \%)$ & $1(2 \%)$ & $<0.001 *$ & & & \\
\hline ACPA +ve. N (\%) & $87(68 \%)$ & $6(24 \%)$ & $2(4 \%)$ & $<0.001 *$ & & & \\
\hline
\end{tabular}

Figure 1 Baseline patient demographics. (A) Baseline classification of patients. Two hundred patients were classified into RA1987 versus UA. RA2010 ACR/EULAR criteria were then applied to patients with UA. Final 3 groups obtained showed 47 patients UA (RA1987-/RA2010-), RA2010 (RA1987-/RA2010+), RA1987 (RA1987+/RA2010+). (B) Demographics according to classification criteria. Data are presented as mean (SD) for continue variables and frequency and percentages for categorical variables. Baseline characteristics between the three groups were compared using the Kruskal-Wallis or Fisher's exact test as appropriate. For post hoc comparison, Dunn tests were run and $p$ value from pairwise comparison reported in the last three columns of the table. ACPA titre, anticitrullinated protein antibody titre (IU/L); ACPA +ve, anticitrullinated protein antibody (>20 IU/L); $\mathrm{CRP}, \mathrm{C}$ reactive protein; DAS28, Disease Activity Score 28 joints; ESR, erythrocyte sedimentation rate; RF titre, rheumatoid factor titre (IU/mL); $\mathrm{RF}+\mathrm{ve}$, rheumatoid factor serum positive (>15IU/L); 28TJC, 28 tender joint count; 28SJC, 28 swollen joint count. 
A

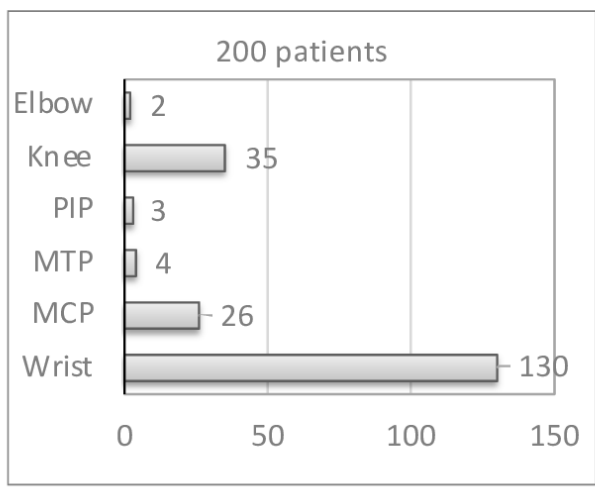

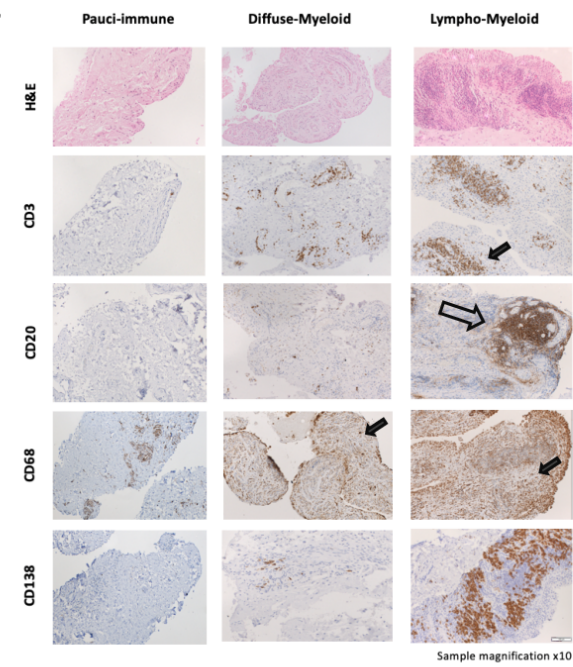

c

\begin{tabular}{|c|c|c|c|c|c|c|c|}
\hline N 166 & $\begin{array}{l}\text { Pauci- } \\
\text { immune } \\
\text { N } 47\end{array}$ & $\begin{array}{l}\text { Diffuse- } \\
\text { Myeloid } \\
\text { N } 57\end{array}$ & $\begin{array}{l}\text { Lympho- } \\
\text { Myeloid } \\
\text { N } 62\end{array}$ & $P$ value & $\begin{array}{c}\text { P-value } \\
\text { (post-hoc) } \\
\text { Lymphoid-M } \\
\text { vs } \\
\text { Pauci-immune }\end{array}$ & $\begin{array}{c}\text { P value } \\
\text { (post-hoc) } \\
\text { Lymphoid-M } \\
\text { vs } \\
\text { Diff-Myeloid }\end{array}$ & $\begin{array}{c}\text { P value } \\
\text { (post-hoc) } \\
\text { Diff-Myeloid } \\
\text { Vs } \\
\text { Pauci-immune }\end{array}$ \\
\hline Age (years). Mean (SD) & $54.93(13.37)$ & $52.64(17.84)$ & $51.90(16.11)$ & 0.51 & & & \\
\hline D. Duration (m). Mean (SD) & $9.21(4.90)$ & $9.30(4.03)$ & $9.54(4.37)$ & 0.98 & & & \\
\hline ESR. Mean (SD) & $33.04(21.68)$ & 28.19 (18.49) & $36.96(19.48)$ & 0.12 & & & \\
\hline CRP. Mean (SD) & 9.55() $13.45)$ & $15.52(14.68)$ & $16.86(12.96)$ & $<0.001 *$ & $<0.001 *$ & 0.06 & $0.013 *$ \\
\hline 28 TJC Mean (SD) & $10.38(8.08)$ & $8.70(6.45)$ & $11.22(7.47)$ & 0.09 & & & \\
\hline 28 SJC. Mean (SD) & $5.70(5.38)$ & $5.96(4.93)$ & $7.75(5.73)$ & 0.054 & & & \\
\hline DAS 28. Mean (SD) & $4.86(1.65)$ & $4.93(1.49)$ & $5.82(1.55)$ & $<0.001 *$ & $0.0012 *$ & $0.002^{*}$ & 1 \\
\hline VAS. Mean (SD) & $50.29(26.87)$ & $53.47(31.33)$ & $61.32(27.94)$ & 0.08 & & & \\
\hline RF +ve. $N(\%)$ & $17(64 \%)$ & $27(53 \%)$ & $40(65 \%)$ & $0.012 *$ & & & \\
\hline ACPA +ve. $N(\%)$ & $15(32 \%)$ & $27(47 \%)$ & $43(70 \%)$ & $0.011 *$ & & & \\
\hline RF titre. Mean (SD) & $10.15(15.40)$ & $20.94(23.95)$ & $23.43(22.74)$ & $0.004 *$ & $0.003 *$ & 0.47 & $0.04 *$ \\
\hline ACPA titre. Mean (SD) & $16.16(28.40)$ & $19.67(24.31)$ & $43.79(104.1)$ & $0.002 *$ & $0.007 *$ & 0.06 & 0.29 \\
\hline
\end{tabular}

\begin{tabular}{|c|c|c|c|c|c|}
\hline N 166 & $\begin{array}{l}1-3 m \\
N=54 \\
N(\%)\end{array}$ & $\begin{array}{l}4-6 \mathrm{~m} \\
\mathrm{~N}=53 \\
\mathrm{~N}(\%)\end{array}$ & $\begin{array}{l}7-9 m \\
N=37 \\
N(\%)\end{array}$ & $\begin{array}{l}10-12 m \\
N=22 \\
N(\%)\end{array}$ & $P$ value \\
\hline Pauci-immune & 19 (34.5\%) & $22(38 \%)$ & $8(28 \%)$ & $8(28.5 \%)$ & \multirow{3}{*}{0.65} \\
\hline Diffuse-Myeloid & $22(40 \%)$ & $17(28 \%)$ & $10(31 \%)$ & $5(18 \%)$ & \\
\hline Lympho-Myeloid & $13(23.5 \%)$ & $14(23 \%)$ & $9(28 \%)$ & $9(32 \%)$ & \\
\hline
\end{tabular}

Figure 2 Patient demographics and disease activity: comparison between pathotypes. (A) Number of biopsy procedures per joint. MCP, metacarpophalangeal; MTP, metatarsophalangeal; PIP, proximal interphalangeal. (B) Representative images of synovial pathotypes. Sections underwent immunohistochemical staining and semiquantitative scoring (0-4) to determine the degree of CD20 +B cells, CD3 +T cells, CD68 +lining) and sublining macrophage and CD138 +plasma cell infiltration. Sections were categorised into three pathotypes: (1) pauci-immune (CD68 SL <2 and or CD3, CD20, CD138<1), (2) diffuse myeloid: (CD68SL >2, CD20<1 and or CD3 >1) and (3) lymphomyeloid: (grade 2-3 CD20 +aggregates, CD20 $>2$ ). Arrow heads indicate positive stain cells. Empty arrows indicate B cell aggregates. (C) Demographic analysis by pathotype. Data are presented as mean and SD for numerical variables and frequency and percentage for categorical variables. Baseline characteristics between the three pathotypes were compared using a Kruskall-Wallis test and Fisher test (RF and ACPA positivity) as appropriate. Post hoc analysis for significant differences using the Dunn test for multiple comparison. A p value of $<0.05$ was considered statistically significant. (D) Pathotype according to disease duration (months) at diagnosis. Absolute values $(\mathrm{N})$ and percentage. A p value of $<0.05$ was considered statistically significant. ACPA titre, anticitrullinated protein antibody titre (IU/L); ACPA +ve, anticitrullinated protein antibody (>20IU/L); CRP, C reactive protein; DAS28, Disease Activity Score 28 joints; ESR, erythrocyte sedimentation rate; RF titre, rheumatoid factor titre (IU/mL); RF +ve, rheumatoid factor serum positive (>15IU/L); $28 \mathrm{TJC}, 28$ tender joint count; 28SJC, 28 swollen joint count. 
A

\begin{tabular}{|l|c|c|c|c|}
\hline N 166 & $\begin{array}{c}\text { RA 1987 } \\
\text { (RA 1987 + / RA 2010+) } \\
\text { N 155 }\end{array}$ & $\begin{array}{c}\text { RA 2010 } \\
\text { (RA 1987 - RA 2010+) } \\
\text { N 23 }\end{array}$ & $\begin{array}{c}\text { UA } \\
\text { (RA1987 - RA2010-) } \\
\text { N 40 }\end{array}$ & p-value \\
\hline $\begin{array}{l}\text { Pauci-immune } \\
47 \text { N (\%) }\end{array}$ & $27(23.5 \%)$ & $6(37.5 \%)$ & $14(40 \%)$ & \multirow{2}{*}{0.10} \\
\cline { 1 - 4 } $\begin{array}{l}\text { Diffuse-Myeloid } \\
57 \text { N (\%) }\end{array}$ & $38(33 \%)$ & $5(31.2 \%)$ & $14(40 \%)$ & \\
\cline { 1 - 4 } $\begin{array}{l}\text { Lympho-Myeloid } \\
62 \mathrm{~N}(\%)\end{array}$ & $50(43.5 \%)$ & $5(31.2 \%)$ & $7(20 \%)$ & \\
\hline
\end{tabular}

B

\begin{tabular}{|c|c|c|c|c|c|c|c|}
\hline N 166 & $\begin{array}{c}\text { RA 1987 } \\
\text { (RA 1987+ + } \\
\text { RA 2010+) } \\
\text { N 155 }\end{array}$ & $\begin{array}{c}\text { RA 2010 } \\
\text { (RA 1987 - / } \\
\text { RA 2010+) } \\
\text { N 23 }\end{array}$ & $\begin{array}{c}\text { UA } \\
\text { (RA1987-/ } \\
\text { RA2010-) } \\
\text { N 40 }\end{array}$ & p-value & $\begin{array}{c}\text { p-value } \\
\text { RA1987-UA }\end{array}$ & $\begin{array}{c}\text { p-value } \\
\text { RA 1987-RA 2010 }\end{array}$ & $\begin{array}{c}\text { p-value } \\
\text { RA 2010-UA }\end{array}$ \\
\hline CD3 & 3.19 & 1.21 & 0.60 & $<0.001^{*}$ & $<0.001^{*}$ & $<0.001^{*}$ & 0.36 \\
\hline CD20 & 2.88 & 0.80 & 0.75 & $<0.001^{*}$ & $<0.001^{*}$ & $<0.001^{*}$ & 0.80 \\
\hline CD68L & 3.60 & 1.86 & 1.34 & $<0.001^{*}$ & $<0.001^{*}$ & $0.0023^{*}$ & 0.18 \\
\hline CD68SL & 3.60 & 2.18 & 1.79 & $0.002^{*}$ & $<0.001^{*}$ & $0.002^{*}$ & 0.24 \\
\hline CD138 & 2.85 & 1.06 & 0.73 & $<0.001^{*}$ & $<0.001^{*}$ & $<0.001^{*}$ & 0.37 \\
\hline Synovitis Score & 6.17 & 3.26 & 3.24 & $<0.001^{*}$ & $<0.001^{*}$ & $0.002^{*}$ & 0.45 \\
\hline
\end{tabular}

\section{RA1987 vs RA2010}

RA1987 subjects 95. RA2010 subjects 12 oH corrected signifiant genes (Breen). 55

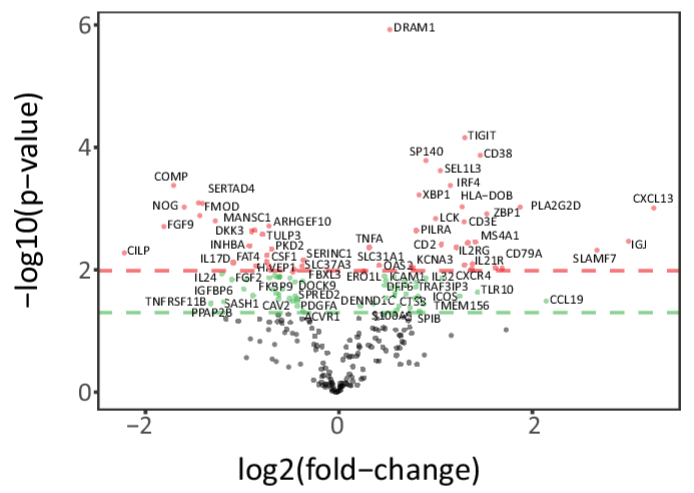

E. RA 2010 vs UA

RA2010 subjects 95. UA subjects 38 Non corrected significant genes (green):

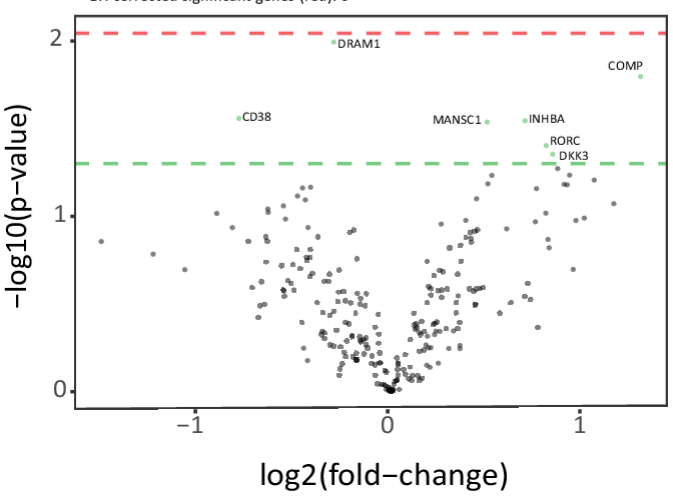

D. RA1987 vs UA

RA1987 subjects 95 . UA subjects 38 Non corrected significant genes (green):

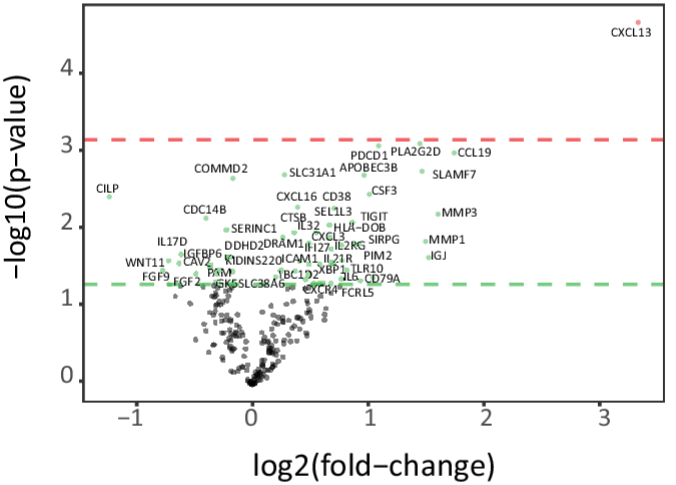
pathotype. Baseline subgroups (RA1987, RA2010 and UA) were compared with pathotype. Fisher test used for analysis. (B) Immune cell infiltration for each clinical subgroup. Kruskal-Wallis test for comparison between three groups. Post hoc analysis for significant differences using the Dunn test for multiple comparison. (C) (C-E) Gene expression analysis for comparison between subgroups. t-Test for comparison and volcano plot for representative image. Positive values represent upregulation and negative values downregulation. Green circles above green horizontal line represents non-corrected for multiple analysis expressed genes between groups. Red circles above red line represents corrected $p$ values (Benjamini-Hochberg (BH) method) for multiple analysis. (C) Volcano plot RA1987 versus RA2010: difference in gene expression between patient fulfilling RA 1987 ACR criteria and RA 2010 ACR/EULAR criteria. (D) Volcano plot RA1987 versus UA: difference in gene expression between patient fulfilling RA 1987 ACR criteria and UA. (E) Volcano plot RA 2010 versus UA: differences in gene expression between patient fulfilling RA 2010 ACR/EULAR criteria and UA. RA, rheumatoid arthritis; UA, undifferentiated arthritis. 


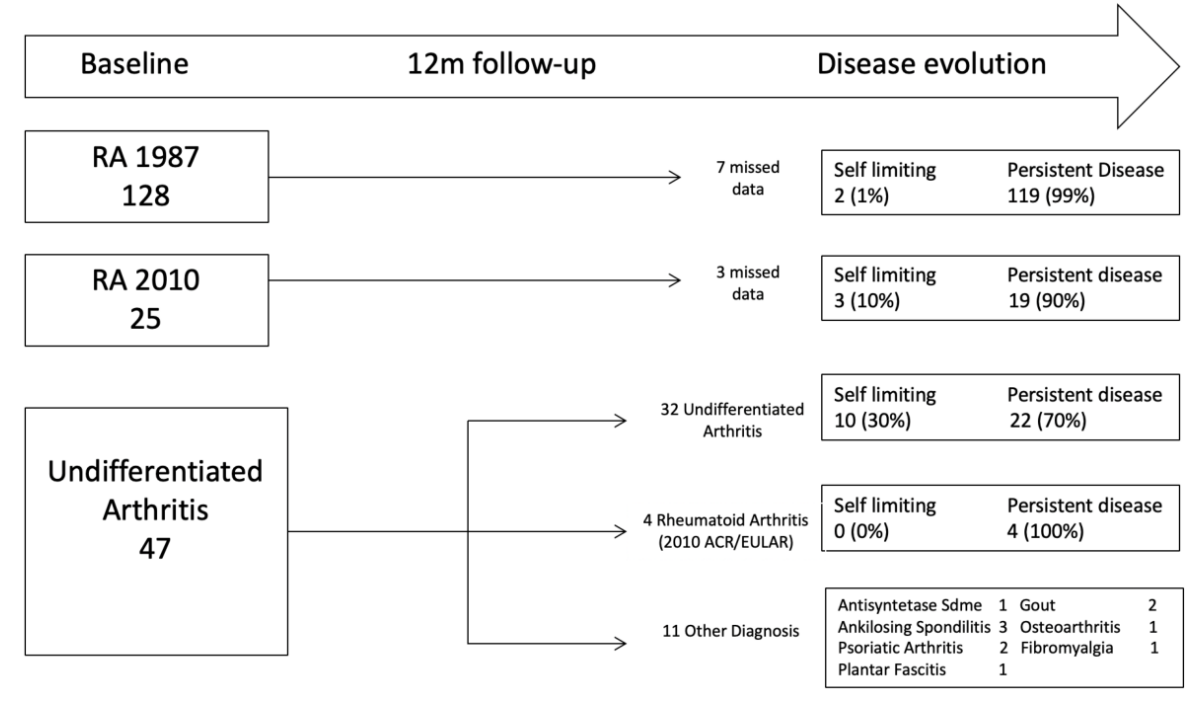

B

\begin{tabular}{|l|c|c|c|c|}
\hline N 179 & $\begin{array}{c}\text { RA 1987 } \\
\text { (RA 1987 + RA 2010+) } \\
\text { N 121 }\end{array}$ & $\begin{array}{c}\text { RA 2010 } \\
\text { (RA 1987 - / RA 2010+) } \\
\text { N 22 }\end{array}$ & $\begin{array}{c}\text { UA } \\
\text { (RA1987 - / RA2010-) } \\
\text { N 36 }\end{array}$ & \multirow{2}{*}{ p-value } \\
\cline { 1 - 3 } $\begin{array}{l}\text { Self limiting } \\
\text { N 15 (\%) }\end{array}$ & $2(13 \%)$ & $3(20 \%)$ & $10(64 \%)$ & \multirow{2}{*}{$<0.001^{*}$} \\
\cline { 1 - 3 } $\begin{array}{l}\text { Persistent disease } \\
\text { N 164 (\%) }\end{array}$ & $119(72 \%)$ & $19(12 \%)$ & $26(16 \%)$ & \\
\hline
\end{tabular}

c

\begin{tabular}{|l|c|c|c|c|}
\hline N 147 & $\begin{array}{c}\text { Pauci-immune } \\
\text { N 41 }\end{array}$ & $\begin{array}{c}\text { Diffuse-Myeloid } \\
\text { N 50 }\end{array}$ & $\begin{array}{c}\text { Lympho-Myeloid } \\
\text { N 56 }\end{array}$ & p-value \\
\hline $\begin{array}{l}\text { Self Limiting } \\
\text { N 11 (\%) }\end{array}$ & $3(27 \%)$ & $6(54 \%)$ & $2(18 \%)$ & \\
\cline { 1 - 3 } $\begin{array}{l}\text { Persistent disease } \\
\text { N 136 (\%) }\end{array}$ & $38(13 \%)$ & $44(32 \%)$ & $54(39 \%)$ & 0.23 \\
\hline
\end{tabular}

Figure 4 Disease evolution. (A) Patient classification after 12-month follow-up. Disease outcome after 12 months of follow-up for each of the initial baseline subgroups (RA1987/RA2010/UA). Disease evolution classified as self-limiting or persistent disease. Other diagnosis as described for those who were reclassified after 1 year form UA cohort. (B) Disease evolution by subgroups. Disease evolution was compared with baseline subgroups (RA1987, RA2010 and UA). Fisher test used for analysis. (C) Disease evolution by pathotype. Disease evolution was compared with pathotype (pauciimmune vs diffuse myeloid vs lymphomyeloid). Fisher test used for analysis. A p value of $<0.05$ was considered statistically significant. RA, rheumatoid arthritis; UA, undifferentiated arthritis.

lympho-myeloid or pauci-immune pathotype (54\% vs $18 \%$ vs 27\%) with SL (figure 4C).

\section{A baseline lympho-myeloid pathotype significantly associates with 12-month requirement for biological therapy}

Patients stratified according to diagnostic group or pathotype were further classified according to 12-month treatment requirement: (1) symptomatic treatment, (2) csDMARDs or (3) biologics \pm csDMARDs. A significantly higher proportion of RA1987 patients required biologic compared with RA2010 and UA $(27.82 \%$ vs $20.83 \%$ vs $10.63 \%)(\mathrm{p}<0.001)$ (figure $5 \mathrm{~A}$ ) and importantly, lympho-myeloid (vs diffuse-myeloid or pauciimmune) pathotype significantly associated with 12-month requirement for biological therapy $(57 \%$ vs $21 \%$ vs $21 \%$ $\mathrm{p}=0.02$ ) (figure $5 \mathrm{~B}$ ).

We then compared expression of the 238 genes in the Nanostring panel between patients requiring biological therapy $(n=34)$ or not $(n=106)$ and found 119 differentially expressed genes. Patients requiring biological therapy had significantly higher differential upregulation of genes regulating $\mathrm{B}$ and $\mathrm{T}$ cell proliferation, differentiation and activation (eg, TNFRSF13C, $C D 79 A, C D 2, C D 3 E$ and $C D 38)$, genes involved in matrix metallopeptidase production/regulation (eg, MMP1 and TIMP1), genes involved in cytokine-mediated cellular activation (TNFA, TRAF3IP3, IFNA1) and osteoclastogenesis inhibition (DEF6). Patients who did not require biological therapy expressed some $\mathrm{B}$ and $\mathrm{T}$ cell regulation genes and $\mathrm{B}$ proliferation markers but mostly markers of fibroblast proliferation and cartilage turnover (figure 5C).

To determine whether disease duration influenced outcome, we segregated patients according to 12-month treatment (biological therapy or not) and further into disease duration quartiles (figure 5D) and demonstrated no significant differences in terms of disease duration at diagnosis. Next, we segregated patients treated with biological therapy $(n=39)$ according to quartiles of disease duration and then synovial pathotype. We found no significant differences in patient number in each quartile $(p=0.3)$ (figure 5E). These results strongly suggest that synovial 
A

\begin{tabular}{|l|c|c|c|c|}
\hline N 186 & $\begin{array}{c}\text { RA 1987 } \\
\text { (RA 1987 + / RA 2010+) } \\
\text { N 115 }\end{array}$ & $\begin{array}{c}\text { RA 2010 } \\
\text { (RA 1987 - RA 2010+) } \\
\text { N 24 }\end{array}$ & $\begin{array}{c}\text { UA } \\
\text { (RA1987 - / RA2010-) } \\
\text { N 47 }\end{array}$ & \multirow{2}{*}{-value } \\
\hline $\begin{array}{l}\text { Symptomatic treatment } \\
\text { N 23 }\end{array}$ & $2(1.7 \%)$ & $4(16.66 \%)$ & $17(36,17 \%)$ & \\
\hline $\begin{array}{l}\text { csDMARDs } \\
\text { N } 121\end{array}$ & $81(70.43 \%)$ & $15(62.50 \%)$ & $25(53,19 \%)$ & \multirow{2}{*}{$<0.001^{*}$} \\
\hline $\begin{array}{l}\text { Biologics +/- csDMARDs } \\
\text { N 42 }\end{array}$ & $32(27.82 \%)$ & $5(20,83 \%)$ & $5(10.63 \%)$ & \\
\hline
\end{tabular}

B

\begin{tabular}{|l|c|c|c|c|}
\hline N 153 & $\begin{array}{c}\text { Pauci-immune } \\
\text { N 44 }\end{array}$ & $\begin{array}{c}\text { Diffuse-Myeloid } \\
\text { N 52 }\end{array}$ & $\begin{array}{c}\text { Lympho-Myeloid } \\
\text { N 57 }\end{array}$ & p-value \\
\hline $\begin{array}{l}\text { Symptomatic Treatment } \\
\text { N } 14\end{array}$ & $6(42 \%)$ & $6(42 \%)$ & $2(14 \%)$ & \\
\hline $\begin{array}{l}\text { csDMARDs } \\
\text { N } 101\end{array}$ & $30(29 \%)$ & $38(37 \%)$ & $33(33 \%)$ & \multirow{2}{*}{$<0.02^{*}$} \\
\cline { 1 - 3 } $\begin{array}{l}\text { Biologics +/- csDMARDs } \\
\text { N 38 }\end{array}$ & $8(21 \%)$ & $8(21 \%)$ & $22(57 \%)$ & \\
\hline
\end{tabular}

C. Biologic vs NonBiologic

Biologic subjects 34. NonBiologic subjects 106 Non corrected significant genes (green): 23
BH corrected significant genes (red): 119

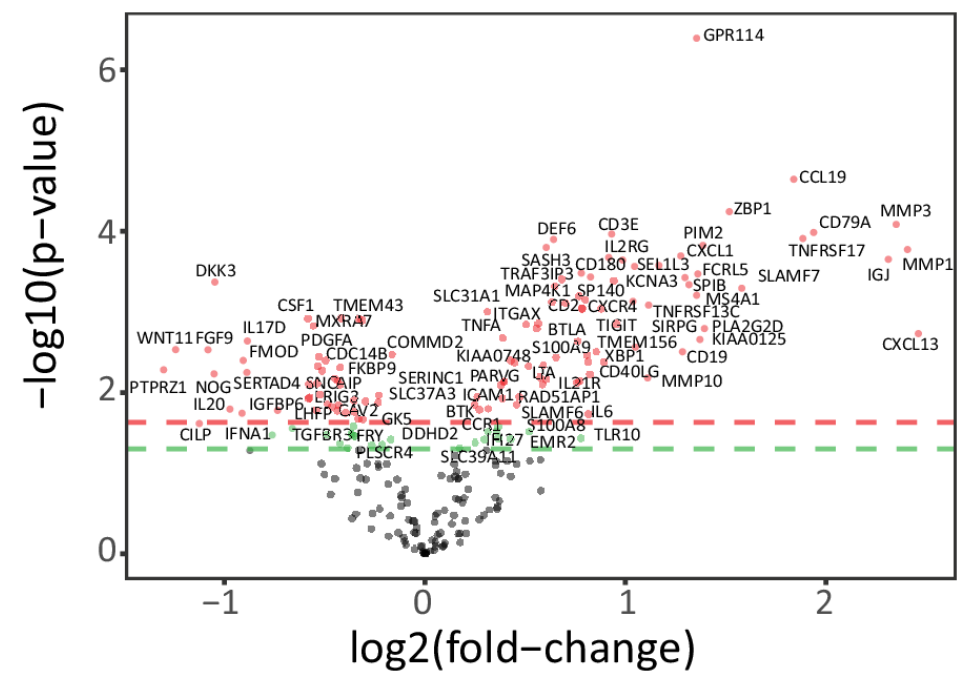

D

\begin{tabular}{|c|c|c|c|c|c|}
\hline N 176 & $\begin{array}{l}1-3 m \\
N 55\end{array}$ & $\begin{array}{l}4-6 m \\
N 61\end{array}$ & $\begin{array}{l}7-9 m \\
N 32\end{array}$ & $\begin{array}{l}10-12 m \\
N 28\end{array}$ & p-value \\
\hline $\begin{array}{l}\text { csDMARDs } \\
\text { n } 137\end{array}$ & $43(30 \%)$ & $43(30 \%)$ & $26(18 \%)$ & $25(17 \%)$ & \multirow{2}{*}{0.23} \\
\hline $\begin{array}{l}\text { Biologics +/- csDMARDs } \\
\text { n } 39\end{array}$ & $12(29 \%)$ & $18(43 \%)$ & $6(14 \%)$ & $3(7 \%)$ & \\
\hline
\end{tabular}

E

\begin{tabular}{|l|c|c|c|c|c|}
\hline \begin{tabular}{l|c|c|c|c|} 
N 39 \\
Biologic cohort
\end{tabular} & $\begin{array}{l}1-3 m \\
\text { N 12 }\end{array}$ & $\begin{array}{l}4-6 m \\
\text { N 18 }\end{array}$ & $\begin{array}{l}7-9 m \\
\text { N 6 }\end{array}$ & $\begin{array}{l}\text { 10-12m } \\
\text { N 3 }\end{array}$ & \multirow{2}{*}{ p-value } \\
\cline { 1 - 4 } Pauciimmune & $1(58 \%)$ & $5(27 \%)$ & $0(0 \%)$ & $2(66 \%)$ & \\
\cline { 1 - 4 } Diffuse - Myeloid & $3(25 \%)$ & $1(5 \%)$ & $3(50 \%)$ & $0(0 \%)$ & \\
\cline { 1 - 4 } Lympho - Myeloid & $7(58 \%)$ & $9(50 \%)$ & $3(50 \%)$ & $1(33 \%)$ & \\
\hline
\end{tabular}

Figure 5 (A) Comparison between diagnostic subgroups and treatment outcome at 12-month follow-up. Treatment required was divided in three groups: (1) no treatment; (2) csDMARDs only, and (3) csDMARDs \pm biologics. Fisher test for analysis. (B) Comparison between pathotype and treatment outcome at 12 months. (C) Gene expression analysis, represented in a volcano plot comparison between patient requiring biologics versus non-biological group. t-Test comparison for gene difference expression between groups. Positive values represent upregulation and negative values downregulation. An adjusted (Benjamini-Hochberg $(\mathrm{BH})$ correction for multiple analysis) $p$ value of $<0.01$ was considered statistically significant, represented as dots above red line. Green dots above green line for gene expression significance when no correction applied for multiple analysis $(p<0.05)$. (D) Treatment outcome according to baseline disease duration. Fisher test for analysis. (E) Pathotype according to baseline disease duration for biological patient cohort. Fisher test for analysis. A p value of $<0.05$ was considered statistically significant unless otherwise stated. RA, rheumatoid arthritis; UA, undifferentiated arthritis. 
pathotype rather than disease duration influences 12-month treatment outcome.

\section{Synovial gene expression signatures enhance the performance of clinical prediction models for biological requirement}

To determine whether baseline clinical and gene expression data could be combined into a model for predicting requirement for biological therapy, we used two complementary approaches: a logistic regression model to identify predictive clinical covariates, and a penalised method based on logistic regression with an L1 regularisation penalty (LASSO) to identify genes improving the clinical model.

Nine baseline clinical covariates were considered as candidates in the regression model: disease duration, ESR, CRP, RF, ACPA, TJC, SJC, DAS28 and pathotype (two categories, lymphomyeloid vs pauci-immune/diffuse-myeloid). Logistic regression models using backward forward and bidirectional stepwise selection resulted in the selection of the same set of clinical covariates: DAS28, pathotype, CRP and TJC. The apparent predictive performance of the model evaluated by AUC was 0.78 (95\% CI 0.70 to 0.87$)$.

Genes were selected to improve the clinical model using logistic regression with an L1 regularisation penalty (LASSO) applied on the four clinical covariates selected by the previous logistic regression and the 119 genes identified as being significantly differentially expressed between the biological and nonbiological groups. Models in which clinical predictors were penalised or subject to forced inclusion were compared. When all predictors were penalised, 11 predictors were retained in the final model and when the clinical covariates were not penalised, 13 predictors were retained (figure 6A). In both the penalised and unpenalised clinical model, the apparent prediction performance was improved (apparent $\mathrm{AUC}=0.89$, 95\% CI 0.83 to 0.95 and $\mathrm{AUC}=0.90,95 \% \mathrm{CI} 0.84$ to 0.95 ) (figure $6 \mathrm{~B}$ ). We additionally performed internal validation to correct the AUC performance measure for overfitting by calculating the optimism of the AUC for each model by bootstrapped sampling with replacement from the original dataset. The optimism corrected AUC was 0.75 for the pure clinical model and 0.81 for the clinical and gene model (LASSO) (figure 6C and D) suggesting that including both clinical covariates and genes in the model results in an improvement of the predictive ability of the model.

\section{DISCUSSION}

These results present a number of novel findings: first, they strongly suggest that patients with early inflammatory arthritis not fulfilling RA1987 criteria display similar clinical, synovial histological and molecular features irrespective of further classification according to RA2010 or UA criteria. Second, these data also suggest that a lympho-myeloid pathotype at disease onset predicts poor outcome with patients subsequently requiring biological therapy irrespective of clinical classification, and finally that the integration of histological and molecular signatures into a clinical prediction model enhances sensitivity/specificity for predicting whether patients will require biological therapy.

To the best of our knowledge, these results emerge from the largest synovial tissue treatment-naïve early arthritis cohort reported to date and support previous data from early RA cohorts suggesting that a synovial immune cell infiltrate characterised by a predominant infiltrate of B cells associates with more active disease $^{18}$ and seropositivity for RF and ACPA. ${ }^{10}$ The results suggest that this effect also extends to patients within the UA cohort. The clinical similarities between RA2010+/RA1987patients and those with UA have been reported previously ${ }^{8}$ and the data presented herein provide a pathophysiological explanation for this with the demonstration of homogeneous synovial cellular and molecular signatures among the two groups. The data show a lower percentage of patients requiring biological therapy in RA2010+/RA1987- group, in line with the expectation that the ACR/EULAR 2010 criteria enable an earlier diagnosis and thus efficacious treatment. However, it is also possible that this group has a milder pathology from the beginning.

Although synovial pathotypes per se do not appear to distinguish between patients at risk of developing PD rather than SL disease, this is not surprising given the early and treat-totarget approach pursued in the study rather than observing untreated natural disease evolution. However, when applying 12-month biological requirement as a prognostic outcome, we demonstrated that patients with a lympho-myeloid pathotype with a dense synovial infiltrate enriched in B cells and significant upregulation of $\mathrm{T} / \mathrm{B}$ cell genes at disease onset predicted requirement for subsequent biological therapy and critically that this was independent of disease duration. These results are consistent with recently published data in early RA which reports that the lympho-myeloid pathotype is associated with highly agressive disease and worse radiographic outcomes. ${ }^{10}$ The current study reinforces these findings demonstrating that, at 12-month follow-up, a significantly higher proportion of patients classified as lympho-myeloid pathotype required biological therapy. The study also calls into question the current dogma surrounding 'an early window of opportunity' for all patients with $\mathrm{RA},{ }^{19-21}$ suggesting that pathotype rather than simply disease duration influences outcome and that intensive therapeutic regimens should be targeted to poor prognostic pathotypes. This notion is supported by the demonstration that the integration of synovial histological and molecular markers into a clinical prediction model for biologics use improves sensitivity/specificity from $78.8 \%$ to $89 \%-90 \%$ independently from disease duration.

Discrepancy with previously reported data suggesting that synovial heterogeneity does not relate to clinical phenotypes ${ }^{9}$ maybe explained by the fact that in our study the majority of biopsies were performed on small joints while in that cohort arthroscopic biopsy was restricted to patients with mainly large joint involvement and, thus, a potential selection bias. ${ }^{22}$ Additionally, the paired histological and molecular data in the largest biopsy-driven early arthritis cohort reported to date ensured internal validation and high classification accuracy.

Our study does have limitations however, for example, the real-life nature of the study did not permit the true evaluation of the natural history of the disease or outcome, as no patients were left untreated and therapy was not actively withdrawn. Also, a treat-to-target approach, treatment escalation and initiation of biological therapy, was determined by treating physicians according to NICE guidelines rather than study protocol.

Within these limitations, our results are robust and suggest that the introduction of the new RA2010 classification criteria brings additional clinical and biological heterogeneity into early patient classification compared with the 1987 criteria with limited ability of RA2010 criteria alone to predict poor outcome. The demonstration that the integration of synovial pathobiological markers into clinical algorithms predicting poor outcome (requirement for biological therapy) independent of disease duration suggests that the 'window of opportunity' is wider than 6 months and early stratification of biological therapies 
A

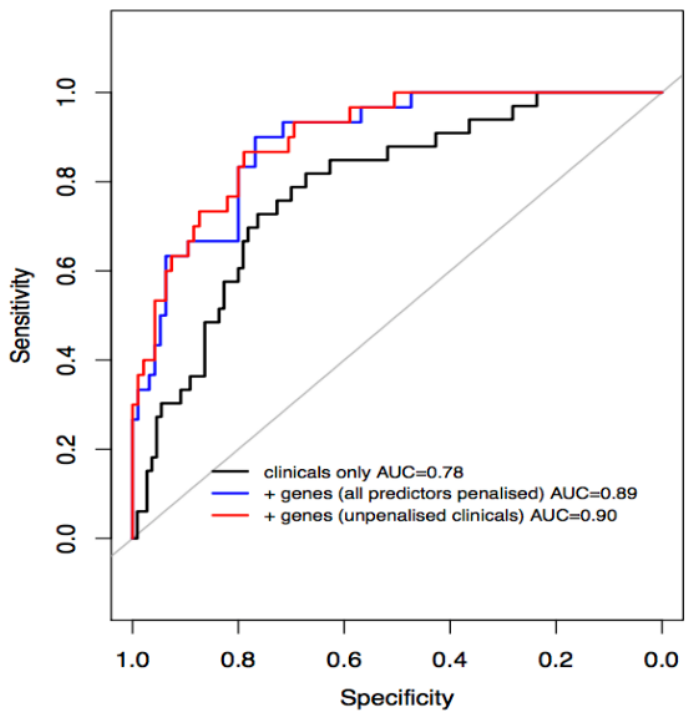

C

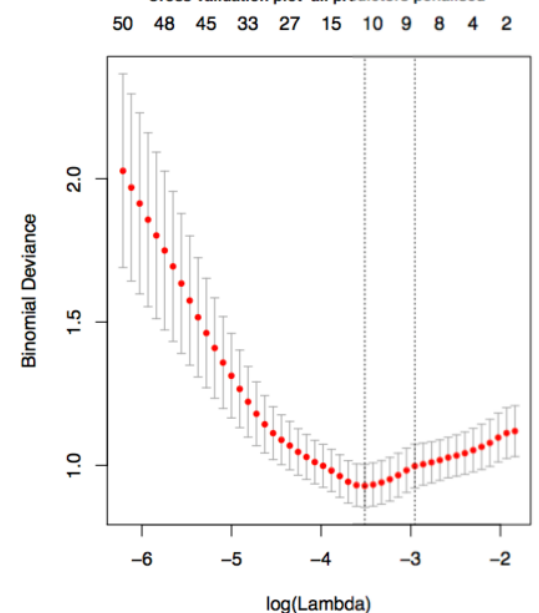

B

\begin{tabular}{|c|c|c|}
\hline & $\begin{array}{c}\text { All predictors } \\
\text { penalised }\end{array}$ & $\begin{array}{c}\text { Unpenalised } \\
\text { clinicals }\end{array}$ \\
\hline (Intercept) & -0.372 & -3.572 \\
\hline Pathotype & & -0.324 \\
\hline CRP & -0.015 & -0.037 \\
\hline TJC & & -0.061 \\
\hline DAS28 & 0.246 & 0.88 \\
\hline GPR114 & 0.242 & 0.295 \\
\hline IL8 & 0.26 & 0.265 \\
\hline CSF1 & -0.08 & -0.034 \\
\hline MMP3 & 0.051 & 0.047 \\
\hline LTB & 0.017 & \\
\hline HIVEP1 & -0.143 & -0.182 \\
\hline IL20 & -0.221 & -0.239 \\
\hline UBASH3A & 0.049 & \\
\hline MMP10 & 0.149 & 0.16 \\
\hline NOG & & -0.038 \\
\hline IFNB1 & & -0.023 \\
\hline
\end{tabular}

D Cross validation plot-unpenalised clinicals $\begin{array}{lllllllllll}50 & 46 & 46 & 42 & 35 & 25 & 18 & 13 & 11 & 6\end{array}$

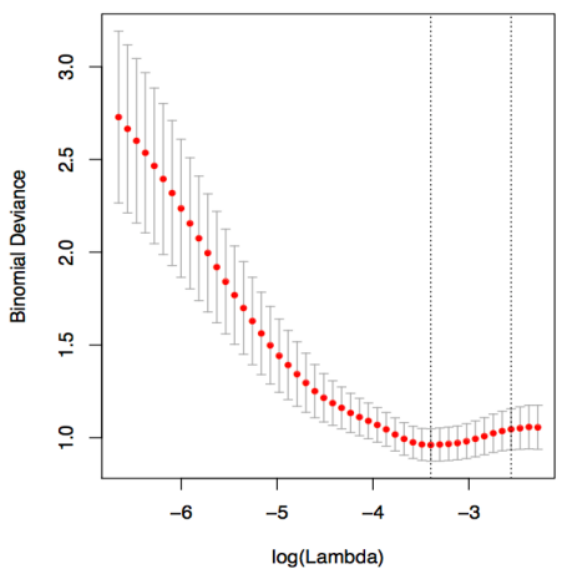

Figure 6 Prediction model. (A) and (B) Identification of clinical and gene expression features predictive of biological therapy use at 1 year. Logistic regression, coupled with backward and stepwise model selection, was applied to baseline clinical parameters against a dependent variable of biological therapy use or not at 12 months to select which clinical covariate contributed the most to the prediction. Selected covariates (119 genes +4 clinical covariates) were entered simultaneously into a logistic model with an L1 regularisation penalty (LASSO) in order to determine the optimal sparse prediction model. A similar predictive performance of the model when clinical was seen when results were penalised (blue-dashed line, A) than when they were not penalised (red-dotted line, A) with a slightly different set of selected covariates (B). (B) Non-zero weights associated with the final variables selected by the LASSO regression. The grey spaces represent the variables that were not selected by the model. (C) and (D) Lambda training curve from the final glmnet fitted model. The red dots represent mean binomial deviance using 10 -fold cross validation. The error bars represent SE of binomial deviance. The vertical dotted lines indicate minimum binomial deviance $\left(\lambda_{\min }\right)$ and a more regularised model for which the binomial deviance error is within one SE of the minimum binomial deviance $\left(\lambda_{1 \mathrm{se}}\right) \cdot \lambda_{\min }$ was selected, corresponding to 11 non-zero coefficients in the final model for the LASSO where clinical were penalised (C) and 13 non-zero coefficients in the final model for the LASSO where clinical were not penalised (D). AUC, area under the receiver operating characteristic curve; CRP, C reactive protein; DAS28, Disease Activity Score 28 joints; TJC, tender joint count.

according to poor prognostic synovial pathobiological subtypes at disease onset may improve the outcome of these patients.

\footnotetext{
Author affiliations

${ }_{1}^{1}$ Centre of Experimental Medicine and Rheumatology, William Harvey Research Institute, London, UK

${ }^{2}$ Centre for Experimental Medicine and Rheumatology, Barts and the London School of Medicine and Dentistry, London, UK

${ }^{3}$ Queen Mary University of London-Charterhouse Square Campus, London, UK

${ }^{4}$ Rheumatology, Barts Health NHS Trust, London, UK

${ }^{5}$ Experimental Medicine and Rheumatology, Queen Marys University of London, London, UK

${ }^{6}$ Biomarker Discovery OMNI, Genentech Research \& Early Development, San Francisco, California, USA
}

${ }^{7}$ ITGR Biomarker Discovery Group, Genentech, South San Francisco, California, USA ${ }^{8}$ Rheumatology and Rheumatology Unit, University of Cagliari and AOU University Clinic of Cagliari, Monserrato, Italy

${ }^{9}$ Institute of Infection and Immunity, Cardiff University School of Medicine, Cardiff, UK

${ }^{10}$ Institute of Inflammation and Ageing, University of Birmingham, Birmingham, UK

${ }^{11}$ Nuffield Department of Orthopaedics, Rheumatology and Musculoskeletal Sciences, University of Oxford, Oxford, UK

${ }^{12}$ Institute of Infection, Immunity and Inflammation, University of Glasgow, Glasgow, UK

${ }^{13}$ Experimental Medicine and Rheumatology, William Harvey Research Institute, London, UK

Acknowledgements The authors thank all study participants. 
Contributors GL-R and FH contributed equally to this manuscript. All authors have contributed to different degrees to patients recruitment and or data generation and or data analysis and or writing the manuscript and or revising data and or manuscript.

Funding Funding for PEAC MRC: Grant code 36661. Funding Infrastructure support to EMR, Arthritis Research UK Experimental Treatment Centre: Grant code 20022.

Competing interests None declared.

Patient consent for publication Not required.

Ethics approval The study received local ethical approval (REC 05/Q0703/198).

Provenance and peer review Not commissioned; externally peer reviewed.

Data availability statement Data are available upon reasonable request.

Open access This is an open access article distributed in accordance with the Creative Commons Attribution 4.0 Unported (CC BY 4.0) license, which permits others to copy, redistribute, remix, transform and build upon this work for any purpose, provided the original work is properly cited, a link to the licence is given, and indication of whether changes were made. See: https://creativecommons.org/ licenses/by/4.0/.

\section{ORCID iDs}

Frances Humby http://orcid.org/0000-0003-1280-9133

Myles Lewis http://orcid.org/0000-0001-9365-5345

Alessandra Nerviani http://orcid.org/0000-0003-4064-4014

Felice Rivellese http://orcid.org/0000-0002-6759-7521

Peter C Taylor http://orcid.org/0000-0001-7766-6167

Costantino Pitzalis http://orcid.org/0000-0003-1326-5051

\section{REFERENCES}

1 Aletaha D, Neogi T, Silman AJ, et al. 2010 rheumatoid arthritis classification criteria: an American College of Rheumatology/European League against rheumatism collaborative initiative. Arthritis Rheum 2010;62:2569-81.

2 Arnett FC, Edworthy SM, Bloch DA, et al. The American rheumatism association 1987 revised criteria for the classification of rheumatoid arthritis. Arthritis \& Rheumatism 1988:31:315-24.

3 van Nies JAB, Brouwer $E$, van Gaalen FA, et al. Improved early identification of arthritis: evaluating the efficacy of Early Arthritis Recognition Clinics. Ann Rheum Dis 2013;72:1295-301.

4 Sakellariou G, Scirè CA, Zambon A, et al. Performance of the 2010 classification criteria for rheumatoid arthritis: a systematic literature review and a meta-analysis. PLoS One 2013:8:1-10.

5 van der Helm-vanMil AHM, le Cessie $\mathrm{S}$, van Dongen $\mathrm{H}$, et al. A prediction rule for disease outcome in patients with recent-onset undifferentiated arthritis: how to guide individual treatment decisions. Arthritis Rheum 2007:56:433-40.
6 Raza K, Falciani F, Curnow SJ, et al. Early rheumatoid arthritis is characterized by a distinct and transient synovial fluid cytokine profile of T cell and stromal cell origin. Arthritis Res Ther 2005; 7:R784-95.

7 Raza K. The Michael Mason prize: early rheumatoid arthritis--the window narrows. Rheumatology 2010;49:406-10.

8 de Hair MJH, Lehmann KA, van de Sande MGH, et al. The clinical picture of rheumatoid arthritis according to the 2010 American College of Rheumatology/ European League against rheumatism criteria: is this still the same disease? Arthritis Rheum 2012:64:389-93.

9 van de Sande MGH, de Hair MJH, Schuller Y, et al. The features of the synovium in early rheumatoid arthritis according to the 2010 ACR/EULAR classification criteria. PLoS One 2012;7:1-7.

10 Humby F, Lewis M, Ramamoorthi N, et al. Synovial cellular and molecular signatures stratify clinical response to cSDMARD therapy and predict radiographic progression in early rheumatoid arthritis patients. Ann Rheum Dis 2019:78:761-72.

11 Kelly S, Humby F, Filer A, et al. Ultrasound-guided synovial biopsy: a safe, welltolerated and reliable technique for obtaining high-quality synovial tissue from both large and small joints in early arthritis patients. Ann Rheum Dis 2015;74:611-7.

12 NICE. Overview | rheumatoid arthritis in adults: management | guidance. Available: https://www.nice.org.uk/guidance/ng100 [Accessed 2 Jul 2019].

13 Krenn V, Morawietz L, Burmester G-R, et al. Synovitis score: discrimination between chronic low-grade and high-grade synovitis. Histopathology 2006;49:358-64.

14 Humby F, Bombardieri M, Manzo A, et al. Ectopic lymphoid structures support ongoing production of class-switched autoantibodies in rheumatoid synovium. PLoS Med 2009; $6: \mathrm{e} 1-75$

15 Dennis G, Holweg CTJ, Kummerfeld SK, et al. Synovial phenotypes in rheumatoid arthritis correlate with response to biologic therapeutics. Arthritis Res Ther 2014;16.

16 Smith GCS, Seaman SR, Wood AM, et al. Correcting for optimistic prediction in small data sets. Am J Epidemiol 2014;180:318-24.

17 Efron B, Tibshirani R. An introduction to the bootstrap. Chapman \& Hall, 1994. https://www.crcpress.com/An-Introduction-to-the-Bootstrap/Efron-Tibshirani/p/book/ 9780412042317

18 Bugatti S, Manzo A, Vitolo B, et al. High expression levels of the B cell chemoattractant CXCL13 in rheumatoid synovium are a marker of severe disease. Rheumatology 2014:53:1886-95.

19 Lard LR, Visser H, Speyer I, et al. Early versus delayed treatment in patients with recent-onset rheumatoid arthritis: comparison of two cohorts who received different treatment strategies. Am J Med 2001;111:446-51.

20 Finckh $\mathrm{A}$, Liang $\mathrm{MH}$, van Herckenrode $\mathrm{CM}$, et al. Long-Term impact of early treatment on radiographic progression in rheumatoid arthritis: a meta-analysis. Arthritis Rheum 2006;55:864-72.

21 Greisen SR, Schelde KK, Rasmussen TK, et al. CXCL13 predicts disease activity in early rheumatoid arthritis and could be an indicator of the therapeutic 'window of opportunity'. Arthritis Res Ther 2014;16.

22 Linn-Rasker SP, van der Helm-van Mil AHM, Breedveld FC, et al. Arthritis of the large joints-in particular, the knee--at first presentation is predictive for a high level of radiological destruction of the small joints in rheumatoid arthritis. Ann Rheum Dis 2007;66:646-50 